\title{
Zur Eröffnung des 31. Jahrgangs der Zeitschrift für öffentliche und gemeinwirtschaftliche Unternehmen
}

Mit diesem Heft 1 geht die Zeitschrift für öffentliche und gemeinwirtschaftliche Unternehmen (ZögU) in ihren 31. Jahrgang. Sie war bislang als wissenschaftliche Zeitschrift zugleich Organ der Gesellschaft für öffentliche Wirtschaft (GÖW), deren Wissenschaftlicher Beirat z. B. hier seine Stellungnahmen veröffentlicht hat. Nunmehr ist der größte Teil der GÖW aufgegangen in den Bundesverband Öffentliche Dienstleistungen (BVÖD) - Deutsche Sektion des CEEP. Die ZögU wird damit zugleich Organ des BVÖD. Dies alles wird zum Anlass genommen, die bereits mit Erscheinen des erstes Heftes des 30. Jahrgangs 2007 erfolgten Veränderungen in der ZögU weiter voran zu treiben.

Wie ersichtlich wird die ZögU nunmehr von einem Herausgeberteam editiert. Angesichts der fachlichen Komplexität des Gegenstandes der ZögU scheint eine derartige multidisziplinäre Herausgeberschaft angemessen. In die Herausgeberschaft sind aus dem Wissenschaftlichen Beirat die Kollegen Thomas Lenk (Leipzig), Holger Mühlenkamp (Speyer) und Dieter Tscheulin (Freiburg i. Br.) und - neu - Johann Christian Pielow (Bochum) berufen worden. Insbesondere der Kollege Pielow folgt somit der Position von Günter Püttner, der viele, viele Jahre in der Herausgeberschaft der ZögU tätig war. Ihm ist an dieser Stelle sehr herzlich zu danken.

Aus dem bisherigen Redaktionsbeirat wird ein Herausgeberbeirat. Dabei verändert sich auch die Präsenz der Wissenschaft innerhalb dieses Herausgeberbeirates. Die Kollegen Helmut Cox (Duisburg - Essen), Werner Wilhelm Engelhardt (Köln) und Christoph Reichard (Potsdam) geben ihre ebenfalls über viele Jahre ausgeübte Beiratstätigkeit auf, ebenso Herr Kollege Dietrich Budäus (Hamburg). Auch Ihnen ist überaus herzlich für die lange Zusammenarbeit zu danken. Kollege Karl Oettle (München) hatte seine Beiratstätigkeit bereits vor einiger Zeit niedergelegt. Ich bin dankbar für die große Leistung dieser Kollegen über viele Jahre hinweg, auch im Wissenschaftlichen Beirat, dem sie allesamt noch erhalten bleiben.

So werden nunmehr aus dem Wissenschaftlichen Beirat Frau Kollegin Christina Schaefer (Berlin) sowie die Kollegen Dietmar Bräunig (Gießen), Ludwig Theuvsen (Göttingen) und Gerold Ambrosius (Siegen) in den Herausgeberbeirat aufgenommen. Seitens des BVÖD kommen neben den bisherigen Beiratsmitgliedern Wilhelm G. Hanss (Vorsitzender der Geschäftsführung der Leipziger Verkehrsbetriebe), Patrick Steinpaß (Direktor des Deutschen Sparkassen- Giroverbandes, Berlin), Reiner Metz (Geschäftsführer ÖPNV des Verbandes Deutscher Verkehrsunternehmen e.V., Köln) und Wolf Leetz (Geschäftsführer des BVÖD, Berlin), Hans-Joachim Reck (Hauptgeschäftsführer des Verbandes kommunaler Unternehmen e.V., Köln) und Dieter Steinkamp (Vertriebsvorstand der Rheinenergie AG, Köln) neu hinzu.

Der sich hier abzeichnende Generationenwechsel, der wie im allgemeinen Lebenszyklus überhaupt wirksam wird, muss ausdrücklich mit der Hervorhebung der Bedeutung der nunmehr zurücktretenden älteren Generation von Kollegen gewürdigt werden. Die ganze Tradition der GÖW und ihres Beirates ist von einer beeindruckenden Generation von 
Universitätsprofessoren getragen worden, die mit der Produktivität des Beirates geradezu personal identisch war. Ohne diese Lebensarbeiten könnte nun die nachfolgende Generation nicht dort weitermachen, wo durch die vorausgegangene das Fundament gelegt und das ganze Gebäude errichtet worden ist. Vielen innigen Dank an diese Kollegen! Ich werde versuchen, im Team die Zeitschrift auf gutem Wege weiter zu entwickeln.

Köln, März 2008

Frank Schulz-Nieswandt

Geschäftsführender Herausgeber und Schriftleiter 